\title{
Efecto del castor en el metabolismo del perifiton y en variables limnológicas de ríos y arroyos fueguinos
}

\author{
Victoria J. García ${ }^{1, \mathbb{D}}$ \& Patricia RodrígueZ ${ }^{1,2}$ \\ ${ }^{1}$ Centro Austral de Investigaciones Científicas (CADIC), CONICET, 9410 Ushuaia, Tierra del Fuego, Argentina. ${ }^{2}$ Universidad \\ Nacional de Tierra del Fuego, Instituto de Ciencias Polares, Ambiente y Recursos Naturales (ICPA).
}

\begin{abstract}
Resumen. El castor norteamericano (Castor canadensis) es una especie invasora en Tierra del Fuego. Es capaz de transformar sistemas lóticos en lénticos por medio de la construcción de diques. Influye sobre la hidrología y la morfología de los ríos, el ciclo de nutrientes, la descomposición, la zona ribereña y sobre las comunidades. El objetivo del presente trabajo fue estudiar el efecto de la actividad del castor sobre variables limnológicas $\mathrm{y}$, en especial, sobre el metabolismo del perifiton (fracciones de masa y clorofila- $a$ ) para evaluar su potencial uso como bioindicador de actividad de castor. Durante el verano se estudiaron seis ríos y arroyos actualmente impactados, y tres arroyos que fueron impactados, pero que ya no poseen actividad. En cada curso de agua se estimaron, río arriba y río abajo de los diques actuales o de donde estuvieron (en el caso de las castoreras abandonadas), las fracciones de masa del perifiton sobre rocas y variables físicas, químicas, hidrológicas y morfológicas. La similitud entre sitios se estudió mediante un análisis de agrupamiento en función de variables morfológicas, hidrológicas y biológicas. Las fracciones de masa del perifiton se analizaron con modelos lineales generalizados mixtos. Río arriba de los diques se diferenciaron los sitios impactados de los no impactados por el castor, así como aquellos que poseían características hidrológicas particulares. Estas diferencias no se observaron río abajo debido a un efecto homogeneizador de la actividad del castor sobre las variables hidrológicas y morfológicas de los tramos impactados. El patrón de la biomasa perifítica no mostró diferencias significativas río abajo de los diques con y sin actividad. Nuestros resultados indican que, en estos arroyos naturalmente heterotróficos, el perifiton no parece ser un buen indicador de la actividad de castor en lo que respecta a las variables de masa y clorofila.
\end{abstract}

[Palabras clave: Castor canadensis, epiliton, fracciones de masa, clorofila perifítica, Patagonia, diques de castor]

\begin{abstract}
AвSTRACT. Beavers' presence affects metabolism of periphyton and limnological variables in Fuegian rivers and streams. The North American beaver (Castor canadensis) is an invasive species in Tierra del Fuego, which creates lentic systems in rivers and streams due to the construction of dams. Beavers influence hydrology and morphology of rivers, nutrient cycle, decomposition, riparian zone, and consequently communities. The aim of this work was to study the effect of beaver dams in subantarctic streams on limnological variables and, specially, on the metabolism (mass fractions and chlorophyll- $a$ ) of periphyton growing on rocks to evaluate its potential use as bioindicator of beaver activity. Six rivers and streams currently impacted and three streams that were impacted, but no longer have activity were studied during summer. Periphyton mass fractions and chlorophyll- $a$ and physical, chemical, hydrological and morphological variables were estimated in each stream, upstream and downstream of current and abandoned dams. Sites similarity was analyzed through a cluster analysis based on morphological, hydrological and biological variables. Periphyton mass fractions upstream and downstream dams were analyzed using generalized linear mixed models. Upstream reaches were clustered regarding beaver activity and stream characteristics. Downstream reaches were similar among them, probably owing to beaver influence, which resulted in a homogenizing effect. Periphyton biomass show no significant differences in downstream reaches regardless beaver activity. Hence, our results indicate that in heterotrophic streams periphyton biomass and chlorophyll are not good bioindicators of beaver activity.
\end{abstract}

[Keywords: Castor canadensis, epilithon, mass fractions, periphyton chlorophyll, Patagonia, beaver ponds]

Editor asociado: Fernando Unrein $\bar{\square}$ victoriajulieta@gmail.com
Recibido: 31 de enero de 2018

Aceptado: 21 de agosto de 2018 


\section{INTRODUCCIÓN}

El castor norteamericano (Castor canadensis) es una especie invasora en la Patagonia sur, introducida en 1946 para fomentar la industria peletera (Daciuk 1978). La ausencia casi total de sus depredadores naturales y la disponibilidad de alimento favoreció la expansión territorial rápida de la población. Esta proliferación generó grandes impactos a escala local (Lizarralde 1993) y constituye la alteración más grande a nivel de paisaje de los bosques y arroyos subantárticos desde la retirada de la última glaciación (10000 D.C.) (Simanonok et al. 2011).

Entre los muchos organismos que modifican su ambiente, los castores influyen tan drásticamente sobre la morfología y biogeoquímica de ríos y arroyos que se los considera "ingenieros de ecosistemas" (Jones et al. 1994; Burchsted et al. 2010). Como tales, pueden transformar sistemas lóticos en lénticos, resultado de la construcción de diques (Lazar et al. 2014). También influyen sobre la hidrología, el ciclo de nutrientes (Correll et al. 2000), la descomposición, la estructura y la dinámica de la zona ribereña, la calidad del agua, los materiales transportados aguas abajo y, en última instancia, sobre las comunidades (Naiman et al. 1988). En función del nivel de actividad, generan a lo largo del cauce una serie de mosaicos que varían entre zonas con flujo de agua libre, agua retenida y hábitats de pradera, como consecuencia de la tala del bosque circundante (Lazar et al. 2014).

En Tierra del Fuego, numerosas investigaciones establecieron el alto grado de impacto que el castor tiene sobre las especies nativas, el hábitat, los ecosistemas y el paisaje (Anderson et al. 2006). Los efectos del castor fueron estudiados sobre diversas comunidades; se encontró que los castores cambian la estructura y la composición de los bosques ribereños de Nothofagus (Anderson et al. 2006; Martínez Pastur et al. 2006), afectan los ensambles de peces nativos y macroinvertebrados acuáticos (Anderson and Rosemond 2007; Moorman et al. 2009) e impactan sobre las redes tróficas (Anderson and Rosemond 2010; Pietrek et al. 2015). Sin embargo, no existen hasta la fecha, estudios realizados en la región acerca del impacto del castor sobre las comunidades algales, recursos basales de todos los organismos del ecosistema acuático.
Entre las comunidades más conspicuas de los cursos de agua lóticos fueguinos se encuentra el perifiton, conformado por algas, hongos, bacterias, protozoos y animales que viven adheridos a un sustrato (Wetzel 1983). En el caso particular de los arroyos fueguinos, el sustrato rocoso es el tipo más frecuente. Al ser una comunidad sésil, no puede evadir los cambios en las condiciones ambientales de su entorno, por lo que constituye un buen indicador de las características de los ecosistemas que habita y de los cambios que en ellos se producen (Lowe and Pan 1996). El estudio de las fracciones de masa del perifiton (i.e., peso seco, cenizas, peso seco libre de cenizas) y clorofila perifítica son buenos indicadores del metabolismo de la comunidad, ya que la proporción de organismos autotróficos y heterotróficos en el ensamble es influenciada por cambios en el ambiente (e.g., disponibilidad de luz [Greenwood and Rosemond 2005], nutrientes [Hill and Fanta 2008], temperatura [Rodríguez and Pizarro 2015] y materia orgánica [Pizarro and Alemanni 2005], entre otros). El objetivo del presente trabajo fue estudiar el efecto de la actividad del castor en arroyos fueguinos sobre variables limnológicas y, especialmente, sobre el metabolismo (fracciones de masa y clorofilaa) del epiliton para evaluar su potencial uso como bioindicadores de disturbio causado por castor.

\section{MAteriales y Métodos}

\section{Área de estudio}

El presente trabajo se llevó a cabo en ríos y arroyos de Tierra del Fuego (52 $39^{\prime}-55^{\circ} 03^{\prime} \mathrm{S}$ y $\left.65^{\circ} 06^{\prime}-68^{\circ} 36^{\prime} \mathrm{O}\right)$, Argentina, localizada en el extremo más austral de América del Sur. Las condiciones insulares y la influencia antártica generan un clima templado frío y húmedo con variaciones locales debido a la orografía de la zona (Pisano 1981). La temperatura media anual es $5^{\circ} \mathrm{C}$, con una media de $10^{\circ} \mathrm{C}$ en verano y $0^{\circ} \mathrm{C}$ en invierno. Las precipitaciones pueden alcanzar los $600 \mathrm{~mm}$ anuales (Barrera et al. 2000; Martínez Pastur et al. 2009; Simanonok et al. 2011). En la isla se pueden distinguir dos grandes áreas con marcadas diferencias en relieve: una región andina montañosa en el sur y una región más plana con suaves ondulaciones en el norte (Lizarralde 1993). La cubierta vegetal está dominada por una mezcla de bosque deciduo de Nothofagus pumilio 
y bosque perenne de Nothofagus betuloides, que alterna con turberas de Sphagnum y vegetación alpina (Moore 1983; Henn et al. 2016). Al norte del Lago Fagnano se extiende un área de transición (denominada ecotono) caracterizada por un mosaico de bosque deciduo de N. pumilio y Nothofagus antarctica que alterna con turberas de Sphagnum y tierras bajas que contienen Carex.

Tres de los arroyos estudiados están localizados en el área andina, en el Parque Nacional Tierra del Fuego (Lapataia, PilotoEnsenada y Piloto-Pampa Alta) (Figura 1). Estos arroyos sufrieron el impacto de la actividad de castores, pero al menos 10 años atrás, castores y castoreras fueron removidos de la zona. Los ríos Esmeralda, Lasifashaj y Pipo, así como el arroyo Hambre, también se encuentran en área andina. Al momento de realizar este estudio, estos ríos estaban afectados por la actividad de los castores y poseían castoreras con actividad. El arroyo Rolito (perteneciente a la cuenca Asturiana) se encuentra en la región del ecotono y también estaba, al momento del estudio, afectado por la actividad de castores desde hacía 6 meses. Finalmente, el arroyo Moat, que también presentaba actividad, se encuentra en la región sureste de la isla, caracterizada por turberas de Sphagnum y Astelia pumilia.

\section{Diseño de muestreo}

En cada sitio se estimó, $50 \mathrm{~m}$ río arriba y $50 \mathrm{~m}$ río abajo de los diques de castor, las fracciones de masa del perifiton junto con parámetros físicos, químicos, hidrológicos y morfológicos, entre los meses de noviembre de 2016 y abril de 2017. Para estudiar las fracciones de masa se colectaron piedras en un área río arriba y en un área río abajo de la castorera, con un marco de PVC de luz de $20 \mathrm{~cm}$ de lado. En el caso de los sitios sin castoreras activas, la ubicación de las castoreras era conocida, de modo que los muestreos se realizaron empleando el mismo diseño.

Para registrar las características morfológicas, en cada sitio (río arriba y río abajo) se midió el ancho mojado y la profundidad a lo largo de diez transectas separadas cada $5 \mathrm{~m}$. En cada transecta se midieron tres profundidades a lo largo de la misma. A partir de estos datos se determinó la profundidad media $(\mathrm{z})$ de la columna de agua y el ancho medio (w). En tres de estas transectas se determinó el caudal (Q) mediante el método de velocidadárea (Gordon et al. 2004). Para determinar la velocidad media (v) se utilizó el método puntual utilizando un flujómetro (G. O. Environmental), calibrado con un molinete hidrométrico Marca Siap modelo 15447.

Las mediciones de $\mathrm{pH}$, temperatura $(\mathrm{T}) \mathrm{y}$ conductividad se realizaron con un sensor de campo marca HANNA 98129 portátil y la concentración de oxígeno disuelto (OD) se midió con un sensor de campo Lutron DO 5510. Se realizaron mediciones de la radiación fotosintéticamente activa (RFA) con un radiómetro Li-250A equipado con un sensor esférico sumergible Li-193SA (Li-Cor, Lincoln) cada $5 \mathrm{~cm}$ en la columna de agua. El coeficiente de atenuación vertical de la RFA, $K_{d}$, se calculó como la pendiente de la relación entre el logaritmo de la irradiancia y la profundidad (Kirk 1994). En cada sitio también se colectaron muestras de agua para determinar las concentraciones de fósforo total (PT) y nitrógeno total (NT) en el agua, la concentración de clorofila- $a$ (cl- $a$ ) fitoplanctónica, de sólidos en suspensión (SS) y la absorbancia a $440 \mathrm{~nm}\left(\mathrm{~A}_{440}\right)$ como indicador de la MOD en el agua (Kirk 2011).

Las muestras biológicas y de agua fueron transportadas en frío y oscuridad para su posterior procesamiento y análisis en el laboratorio. Para determinar la cl-a fitoplanctónica se filtró un volumen conocido de agua con filtros de fibra de vidrio Whatman GF/F. El filtro con el material retenido se guardó en oscuridad a $-20{ }^{\circ} \mathrm{C}$ hasta la determinación del pigmento. Para determinar las fracciones de masa del perifiton, peso seco (PS), peso seco libre de cenizas (PSLC) y cenizas (PC), los sustratos se rasparon con un cepillo de cerda suave y el material raspado se resuspendió en un volumen conocido de agua destilada. Una fracción de volumen conocido de la resuspensión fue filtrada por filtros Whatman GF/F pre-pesados y pre-muflados $\left(500{ }^{\circ} \mathrm{C}, 3 \mathrm{~h}\right)$. Los filtros con el material retenido fueron secados en estufa a $60^{\circ} \mathrm{C}$ hasta peso constante para estimar el PS y posteriormente calcinados en una mufla a 500 ${ }^{\circ} \mathrm{C}$ durante $3 \mathrm{~h}$ para estimar el PC, y el PSLC, por diferencia con el PS obtenido previamente. La concentración de cl-a perifítica se determinó a partir del filtrado por filtros Whatman GF/F de un volumen conocido de la resuspensión. Los filtros fueron almacenados durante al menos $24 \mathrm{~h} \mathrm{a}-20^{\circ} \mathrm{C}$. La extracción de cl- $a$ tanto perifítica como fitoplanctónica se realizó con etanol caliente $\left(60-70{ }^{\circ} \mathrm{C}\right)$ y la concentración de cl- $a$, corregida por feopigmentos, se determinó por espectrofotometría, antes y 
después de acidificar $(\mathrm{HCl} 0.1 \mathrm{~N})$ utilizando un espectrofotómetro Cintra (Jespersen and Christoffersen 1987). A partir de estos datos se calculó el índice autotrófico (IA) como el cociente entre el PSLC y la concentración de cl-a perifítica. Este índice permite evaluar si la composición de la comunidad es más autotrófica o heterotrófica: valores superiores a 200 indican una alta proporción de organismos heterotróficos, no clorofílicos y detritus orgánico (APHA 2005; Lowe and Pan 1996). La comunidad también se clasificó según Lakatos (1989) en referencia a su PS, contenido de cenizas y concentración de cl- $a$.

Las concentraciones de PT y NT se determinaron como fosfato $\left(\mathrm{P}_{-} \mathrm{PO}_{4}\right)$ y nitrato $\left(\mathrm{N}-\mathrm{NO}_{3}\right)$ luego de una oxidación conjunta con ácido bórico e hidróxido de sodio siguiendo la metodología propuesta por Valderrama (1981). La concentración de $\mathrm{P}_{-} \mathrm{PO}_{4}$ en las muestras oxidadas se determinó mediante el método del ácido ascórbico y la de $\mathrm{N}-\mathrm{NO}_{3}$ con el método de diazotización, previa reducción con cadmio (APHA 2005), empleando reactivos y espectrofotómetro Hach. Para determinar los SS se filtró un volumen de agua conocido mediante filtros de fibra de vidrio de $1 \mu \mathrm{m}$ de tamaño de poro pre-secados y pre-pesados. Los filtros con el material retenido se secaron en estufa a $60^{\circ} \mathrm{C}$ hasta peso constante y la concentración de SS se obtuvo por diferencia de pesos (APHA 2005). La $\mathrm{A}_{440}$ se determinó en agua previamente filtrada por filtros Whatman GF/F en una cubeta de cuarzo de $1 \mathrm{~cm}$ de paso óptico y con un espectrofotómetro Cintra.

\section{Análisis estadístico}

Los datos se presentan como la media \pm el desvío estándar (DS). La similitud entre sitios de estudio, tanto río arriba como río abajo de los diques de castor, se analizó, en primer lugar, mediante un análisis de agrupamiento por conglomerados en función de variables morfológicas, hidrológicas y biológicas. Para dicho análisis se utilizó la distancia Euclídea y el algoritmo de encadenamiento promedio o método de agrupamiento de pares no ponderado utilizando una media aritmética (UPGMA por sus siglas en inglés) (Sokal and Michener 1958) y se incluyeron las variables para las cuales se contó con mayor número de datos: PS del perifiton, cl- $a$ perifítica y fitoplanctónica, $\mathrm{K}_{\mathrm{d}}$, SS, v y w. Dado que en el sitio río abajo del arroyo Moat no se encontró epiliton, el mismo no fue incluído en el agrupamiento de los sitios río abajo. Para conocer la importancia relativa de las variables utilizadas en el agrupamiento por

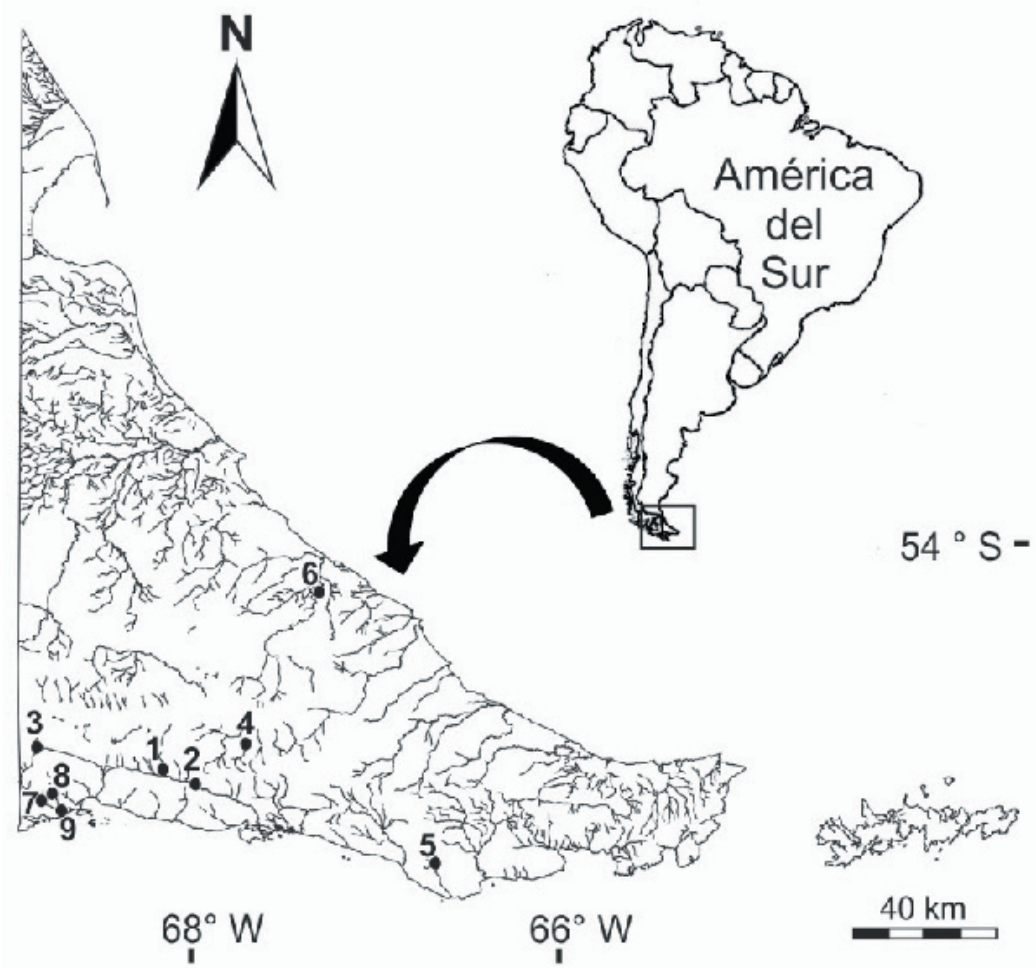

Figura 1. Mapa de Tierra del Fuego con la ubicación de los ríos y arroyos estudiados. 1: Río Esmeralda, 2: Río Lasifashaj, 3: Río Pipo, 4: arroyo Hambre, 5: arroyo Moat, 6: arroyo Rolito, 7: arroyo Lapataia, 8: arroyo Piloto-Ensenada, 9: arroyo Piloto-Pampa Alta.

Figure 1. Map of Tierra del Fuego showing the location of the studied rivers and streams. 1: Esmeralda River, 2: Lasifashaj River, 3: Pipo River, 4: Hambre stream, 5: Moat stream, 6: Rolito stream, 7: Lapataia stream, 8: Piloto-Ensenada stream, 9: Piloto-Pampa Alta stream. 
conglomerados se realizó, a posteriori, un análisis de componentes principales (ACP) que incluyó las mismas variables biológicas y ambientales utilizadas en el agrupamiento por conglomerados.

Los resultados de las fracciones de masas del perifiton (PS, PC, PSLC y cl-a) río arriba y río abajo fueron analizados con modelos lineales generalizados mixtos (del inglés, generalized linear mixed models [GLMM]), con el programa estadístico $\mathrm{R}$ y el paquete de datos de GLMM (Zuur et al. 2009), en un entorno de InfoStat. Se consideró al tramo (río arriba o río abajo) y a la actividad del castor (con y sin) como factores fijos y a los sitios de muestreo (río/arroyo, unidad experimental) como factor aleatorio. Para realizar dichos análisis se ajustaron los modelos con distinta homocedasticidad y mediante el criterio de verosimilitud AIC (del inglés, akaike information criteria) e inspección gráfica de su distribución residual, se eligió el mejor modelo (Balzarini et al. 2008). Se estudiaron los efectos principales independientemente. En caso de detectarse diferencias significativas, se realizó un análisis de LSD Fisher a posteriori de comparación de medias. La distribución normal de los residuos fue analizada, para todos los análisis, con la prueba estadística de normalidad de Shapiro-Wilks. Las posibles relaciones entre las variables biológicas, físicas y químicas río arriba y río abajo se analizaron con correlaciones de Spearman. Las variables incluidas en este análisis fueron: $\mathrm{K}_{\mathrm{d}}, \mathrm{A}_{440}, \mathrm{PT}$, $\mathrm{NT}$, cl-a fitoplanctónica, SS y v media $(\mathrm{n}=9)$, cl-a perifítica y PS del perifiton $(\mathrm{n}=9$ río arriba y $\mathrm{n}=8$ río abajo), y PSLC del perifiton, IA del perifiton y PC del perifiton ( $\mathrm{n}=8$ río arriba y $n=7$ río abajo). Para todos los análisis estadísticos se utilizó el programa InfoStat versión 2017 (Di Rienzo et al. 2017) y el nivel de significancia fue fijado en 0.05 .

\section{Resultados}

Características morfológicas, hidrológicas, físicas y químicas

En general, los sitios con actividad de castor presentaron mayores valores de temperatura del agua, w , z, v y Q, tanto río arriba como río abajo de los diques de castor (Tabla 1). El $\mathrm{K}_{\mathrm{d}^{\prime}}$ la concentración de SS y la MOD (estimada como $\mathrm{A}_{440}$ ) también fueron mayores en los sitios con actividad y, en el caso de los SS y la MOD, disminuyeron en los tramos río abajo. El NT en los sitios con actividad fue mayor río arriba. El PT fue mayor en los sitios con actividad, en especial en los tramos río abajo (Tabla 1).

Los sitios sin actividad presentaron mayores valores de conductividad y OD (especialmente río abajo). Si bien la concentración de SS y la MOD fueron menores que en los sitios con actividad, las mismas aumentaron río abajo

Tabla 1. Variables morfológicas, hidráulicas, físicas, químicas y biológicas medidas en los ríos y arroyos estudiados. Las variables se presentan como las medias \pm DS de los sitios con y sin actividad, río arriba y río abajo de los diques de castor.

Table 1. Morphological, hydraulic, physical, chemical and biological variables measured in the streams and rivers studied. Variables are presented as means \pm SD of sites with and without activity, upstream and downstream of beaver ponds.

\begin{tabular}{|c|c|c|c|c|}
\hline & \multicolumn{2}{|l|}{ Con actividad } & \multicolumn{2}{|c|}{ Sin actividad } \\
\hline & Río arriba & Río abajo & Río arriba & Río abajo \\
\hline w medio (m) & $6.56 \pm 3.83$ & $7.12 \pm 6.55$ & $1.36 \pm 0.53$ & $1.36 \pm 0.28$ \\
\hline $\mathrm{z}$ media $(\mathrm{m})$ & $0.30 \pm 0.09$ & $0.28 \pm 0.13$ & $0.09 \pm 0.03$ & $0.13 \pm 0.08$ \\
\hline $\mathrm{v}$ media $(\mathrm{m} / \mathrm{s})$ & $0.34 \pm 0.14$ & $0.41 \pm 0.18$ & $0.31 \pm 0.07$ & $0.35 \pm 0.08$ \\
\hline $\mathrm{Q}\left(\mathrm{m}^{3} / \mathrm{s}\right)$ & $1.01 \pm 1.48$ & $1.05 \pm 1.08$ & $0.07 \pm 0.10$ & $0.04 \pm 0.02$ \\
\hline $\mathrm{pH}$ & $7.34 \pm 0.69$ & $7.38 \pm 0.59$ & $7.74 \pm 0.31$ & $7.70 \pm 0.24$ \\
\hline Conductividad $(\mu \mathrm{S} / \mathrm{cm})$ & $112 \pm 59$ & $112 \pm 60$ & $162 \pm 9$ & $161 \pm 3$ \\
\hline $\mathrm{OD}(\mathrm{mg} / \mathrm{L})$ & $10.11 \pm 1.94$ & $10.98 \pm 0.67$ & $11.63 \pm 0.75$ & $12.67 \pm 1.12$ \\
\hline $\mathrm{T}$ agua $\left({ }^{\circ} \mathrm{C}\right)$ & $10.53 \pm 1.76$ & $10.08 \pm 2.97$ & $5.07 \pm 3.25$ & $5.30 \pm 2.69$ \\
\hline $\mathrm{K}_{\mathrm{d}}(1 / \mathrm{m})$ & $4.73 \pm 4.85$ & $4.42 \pm 5.35$ & $1.64 \pm 0.61$ & $1.44 \pm 0.88$ \\
\hline $\mathrm{SS}(\mathrm{mg} / \mathrm{L})$ & $8.82 \pm 12.98$ & $4.71 \pm 2.45$ & $1.84 \pm 2.45$ & $3.75 \pm 4.83$ \\
\hline $\mathrm{A}_{440}(1 / \mathrm{m})$ & $4.95 \pm 9.02$ & $3.72 \pm 6.32$ & $0.59 \pm 0.65$ & $1.02 \pm 1.23$ \\
\hline $\mathrm{NT}(\mathrm{mg} / \mathrm{L})$ & $2.08 \pm 1.07$ & $1.48 \pm 0.26$ & $1.85 \pm 0.18$ & $2.00 \pm 0.35$ \\
\hline PT (mg/L) & $0.28 \pm 0.18$ & $0.34 \pm 0.36$ & $0.10 \pm 0$ & $0.18 \pm 0.10$ \\
\hline cl- $a$ fitoplancton $(\mu \mathrm{g} / \mathrm{L})$ & $1.04 \pm 2.17$ & $0.27 \pm 0.42$ & $0.26 \pm 0.15$ & $0.19 \pm 0.19$ \\
\hline $\mathrm{cl}-a$ perifiton $\left(\mathrm{mg} / \mathrm{m}^{2}\right)$ & $0.25 \pm 0.39$ & $0.14 \pm 0.12$ & $0.39 \pm 0.21$ & $0.30 \pm 0.31$ \\
\hline PS perifiton $\left(\mathrm{g} / \mathrm{m}^{2}\right)$ & $4.33 \pm 4.33$ & $2.07 \pm 1.10$ & $10.70 \pm 6.48$ & $2.55 \pm 0.59$ \\
\hline PSLC perifiton $\left(\mathrm{g} / \mathrm{m}^{2}\right)$ & $1.82 \pm 2.74$ & $0.60 \pm 0.31$ & $1.71 \pm 0.84$ & $0.78 \pm 0.23$ \\
\hline $\mathrm{PC}\left(\mathrm{g} / \mathrm{m}^{2}\right)$ & $2.72 \pm 1.98$ & $1.82 \pm 0.97$ & $8.99 \pm 5.67$ & $1.76 \pm 0.44$ \\
\hline IA perifiton & $11986 \pm 13687$ & $12233 \pm 13211$ & $4913 \pm 3243$ & $4434 \pm 2742$ \\
\hline
\end{tabular}


(Tabla 1). El pH fue similar entre todos los sitios con valores cercanos a la neutralidad.

\section{Variables biológicas}

Los sitios con actividad presentaron mayores valores de cl-a fitoplanctónica (aunque esta diferencia no resultó estadísticamente significativa). A su vez, en estos sitios la cla fitoplanctónica fue más variable río arriba (coeficiente de variación, $\mathrm{cv}=2.09$ ) que río abajo $(\mathrm{cv}=1.55)$ (Tabla 1). Los sitios sin actividad presentaron mayores concentraciones de cla perifítica, aunque esta diferencia tampoco resultó estadísticamente significativa, tanto en el nivel de actividad o como en el de tramo. El PS del perifiton fue mayor en los sitios sin actividad (aunque no estadísticamente significativo).

Al comparar entre tramos, si bien no se detectaron diferencias significativas, tanto el PS como el PSLC del perifiton, así como el PC fueron mayores en los tramos río arriba, independientemente de la actividad de castor. En general, en todas las fracciones de masa del perifiton se observó una disminución río abajo, independientemente de la presencia de castor (Tabla 1).

Según la clasificación de Lakatos (1989) en relación con el peso seco, en todos los sitios (tanto con actividad como sin) y en ambos tramos (arriba y abajo), el perifiton puede clasificarse como perifiton de tipo III, es decir, de baja biomasa. Según el porcentaje de cenizas, el perifiton resultó orgánico para todos los sitios, mientras que en función del porcentaje de cl- $a$ en el peso seco resultó heterotrófico en todos los sitios. El IA también evidenció una comunidad perifítica altamente heterotrófica, ya que fue alto en todos los sitios. Así mismo, cabe destacar que en los sitios con actividad el IA fue más del doble que en los sitios sin actividad (Tabla 1).

\section{Análisis de agrupamiento por conglomerados}

El análisis de agrupamiento por conglomerados de los sitios río arriba evidenció dos grupos claramente distinguibles entre los que se agruparon los sitios Pipo, Hambre, Lasifashaj y Esmeralda, por un lado, y los arroyos sin actividad actual (Lapataia, Piloto-Pampa Alta y Piloto-Ensenada), por otro (Figura 2). Los arroyos Rolito y Moat no formaron parte de estos grupos, separándose del resto a una distancia mayor.

En el análisis de agrupamiento de los sitios río abajo, los patrones de ordenamiento que se distinguían entre los sitios río arriba se pierden (Figura 3). El único grupo que se distingue en el ordenamiento incluye sitios con y sin actividad (río Lasifashaj y arroyos Lapataia y Hambre).

\section{Análisis de componentes principales (ACP)}

En el ACP río arriba se observó que los dos primeros componentes explican $63.8 \%$ de la variabilidad total y que el componente 2 separa dos grupos la mayoría de los sitios estudiados. Por un lado, se encuentran los

Río Arriba

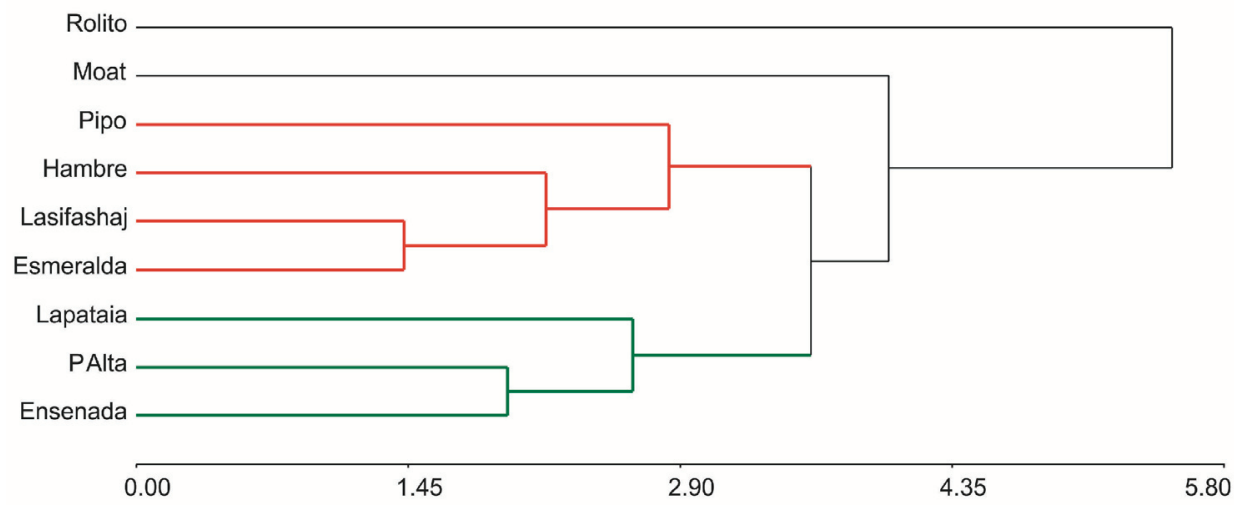

Figura 2. Dendrograma que describe el agrupamiento de los sitios río arriba en función de las variables PS del perifiton, cl-a perifítica y fitoplanctónica, $\mathrm{K}_{\mathrm{d}}$ SS, T del agua, v media y w medio. Se utilizaron la distancia Euclídea y el algoritmo UPGMA como método de ligamiento.

Figure 2. Cluster analysis of upstream sites. Variables included: perifiton's DW, periphyton and phytoplankton's chl- $a$, $\mathrm{K}_{\mathrm{d}^{\prime}}$ SS, water T, v and w. The Euclidean distance and the UPGMA algorithm were used as linkage method. 
sitios Hambre, Lasifashaj, Moat, Esmeralda y Pipo (i.e., los sitios con actividad, a excepción del arroyo Rolito); por el otro, los arroyos sin actividad actual (Piloto-Pampa Alta, PilotoEnsenada y Lapataia) (Figura 4). La variable de mayor peso en el agrupamiento de los sitios con actividad fue el ancho $(\mathrm{w})$ medio, variable con alto peso negativo en el componente 1 (autovector e1=-0.39) y positivo el componente 2 (autovector $\mathrm{e} 2=0.49$ ). Los sitios sin actividad se agruparon por poseer menor temperatura del agua, variable con alto valor positivo en el componente 2 (autovector e $2=0.58$ ) y mayor PS del perifiton, con alto peso negativo en el componente 2 (autovector e2=-0.32). El arroyo Rolito no formó parte de ningún grupo separándose del resto de los arroyos y se asoció a mayores valores de las variables SS, cl- $a$ fitoplactónica y cl-a perifítica, con alto peso positivo en el componente 1 (autovectores $\mathrm{e} 1=0.49,0.52$ y 0.56 , respectivamente).

En el ACP río abajo, se observó que los dos primeros componentes explican $61 \%$ de la variabilidad total. Sin embargo, al igual que en el agrupamiento por conglomerados, los grupos que se distinguían entre los sitios de estudio río arriba ya no se distinguen en este análisis (Figura 5). El único grupo que se forma y se separa principalmente por la componente

\section{Río Abajo}

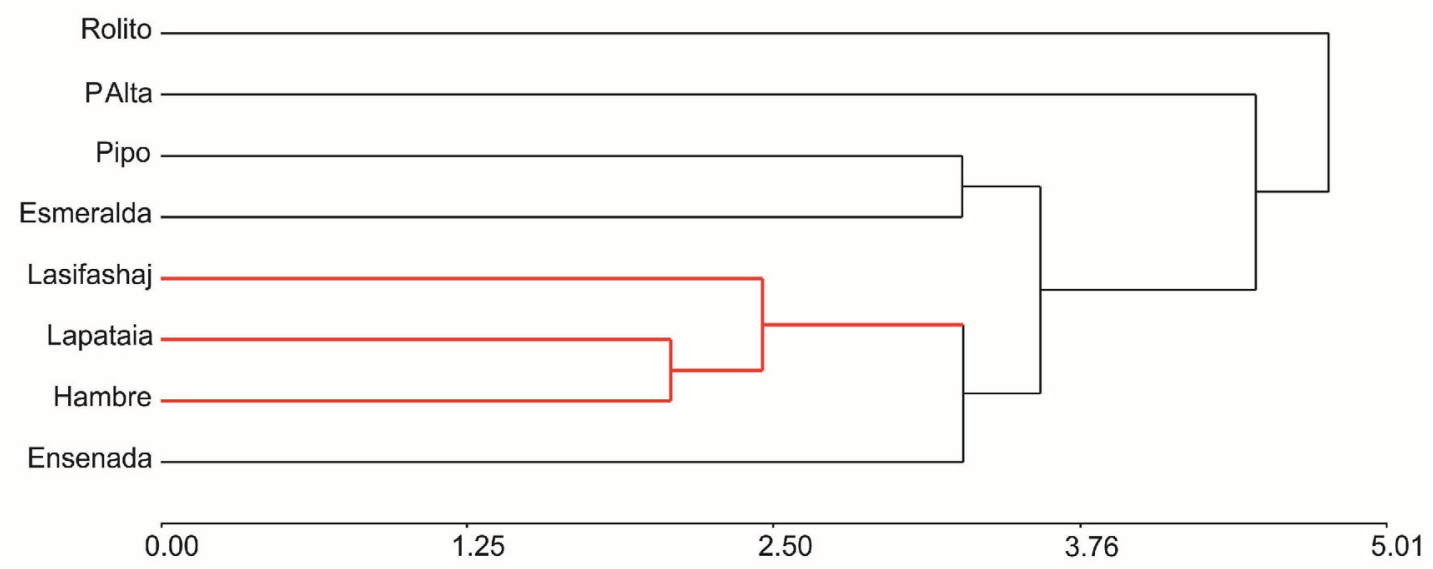

Figura 3. Dendrograma que describe el agrupamiento de los sitios río abajo en función de las variables PS del perifiton, cl-a perifítica y fitoplanctónica, $\mathrm{K}_{\mathrm{d}}$, SS, T del agua, v media y w medio. Se utilizó la distancia Euclídea y el algoritmo UPGMA como método de ligamiento.

Figure 3. Cluster analysis of downstream sites. Variables included: perifiton's DW, periphyton and phytoplankton's $\mathrm{cl}-a, \mathrm{~K}_{\mathrm{d}^{\prime}}$ SS, water T, v and w. The Euclidean distance and the UPGMA algorithm were used as linkage method.

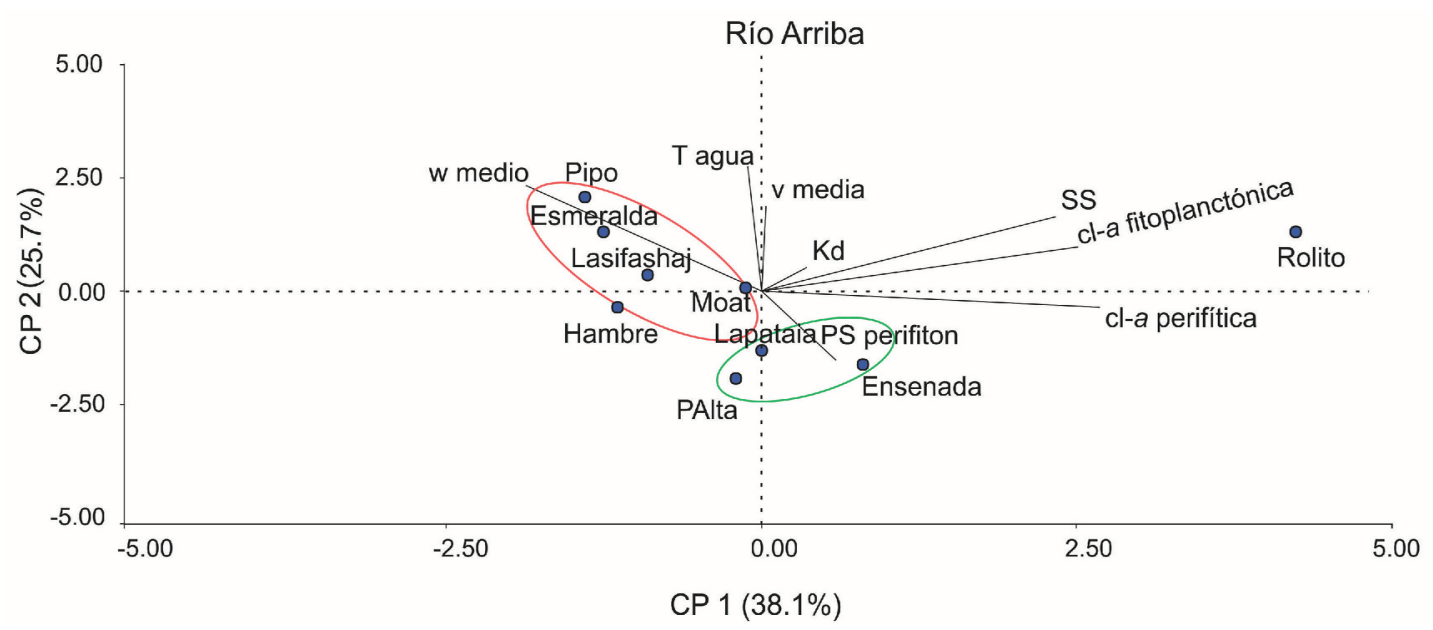

Figura 4. Análisis de componentes principales para los sitios río arriba en función de las variables PS del perifiton, cl-a perifítica y fitoplanctónica, $\mathrm{K}_{\mathrm{d}^{\prime}} \mathrm{SS}$, T del agua, v media y w medio.

Figure 4. Principal components analysis of upstream sites. Variables included: perifiton's DW, periphyton and phytoplankton's cl- $a, \mathrm{~K}_{\mathrm{d}}$, SS, water T, v and w. 


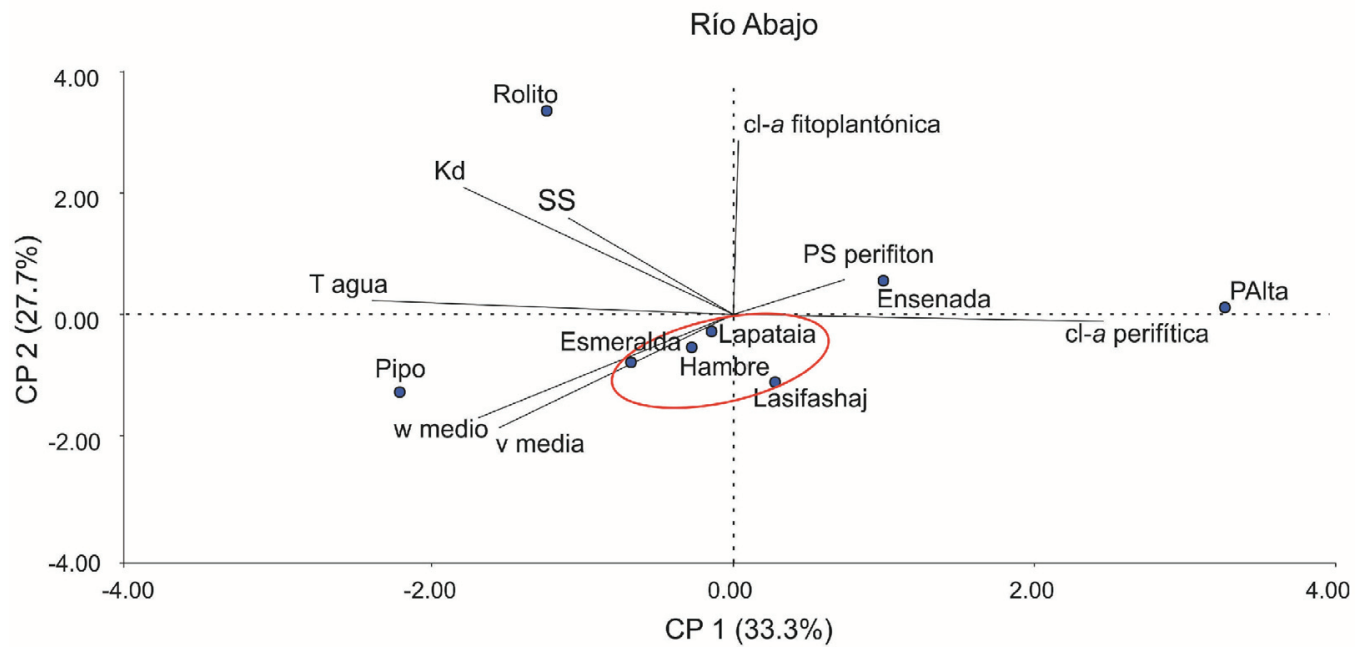

Figura 5. Análisis de componentes principales para los sitios río abajo en función de las variables PS del perifiton, cl- $a$ perifítica y fitoplanctónica, $\mathrm{K}_{\mathrm{d}} \mathrm{SS}$, T del agua, v media y w medio.

Figure 5. Principal components analysis of downstream sites. Variables included: perifiton's DW, periphyton and phytoplankton's cl- $a, \mathrm{~K}_{\mathrm{d}}$, SS, water T, v and w.

1 , incluye arroyos con y sin actividad (ríos Esmeralda y Lasifashaj y arroyos Lapataia y Hambre). Estos sitios se agruparon principalmente debido a altos valores de $\mathrm{w}$ medio y v media (con autovectores de -0.36 y -0.33 , respectivamente, en la componente 1$)$.

Tabla 2. Correlaciones de Spearman entre las variables medidas río arriba y río abajo. Se muestran el coeficiente de correlación de Spearman y el valor de $P$ de las correlaciones significativas. Para todas las correlaciones río arriba, $n=9$ excepto en para la variable IA $(n=8)$. Para todas las correlaciones río abajo, $\mathrm{n}=9$ excepto para la variable IA $(\mathrm{n}=7)$.

Table 2. Spearman correlations between variables studied in the upstream sites. Spearman correlation coefficient and $P$-values of significant correlations are shown. For all upstream variables $n=9$ except for AI $(n=8)$. For all downstream variables $n=9$ except for $\mathrm{AI}(n=7)$.

\begin{tabular}{|c|c|c|c|c|}
\hline Tramo & Variable 1 & Variable 2 & r Spearman & $P$-valor \\
\hline \multirow[t]{7}{*}{$\begin{array}{l}\text { Río } \\
\text { arriba }\end{array}$} & $\mathrm{K}_{\mathrm{d}}(1 / \mathrm{m})$ & PT (mg/L) & 0.86 & 0.003 \\
\hline & $\mathrm{K}_{\mathrm{d}}(1 / \mathrm{m})$ & $\mathrm{A}_{440}(1 / \mathrm{m})$ & 0.85 & 0.003 \\
\hline & NT (mg/L) & $\begin{array}{l}\text { cl-a perifiton } \\
\left(\mathrm{mg} / \mathrm{m}^{2}\right)\end{array}$ & 0.78 & 0.014 \\
\hline & $\mathrm{NT}(\mathrm{mg} / \mathrm{L})$ & $\begin{array}{l}\text { PS perifiton } \\
\left(\mathrm{g} / \mathrm{m}^{2}\right)\end{array}$ & 0.79 & 0.01 \\
\hline & PT (mg/L) & $\mathrm{A}_{440}(1 / \mathrm{m})$ & 0.73 & 0.025 \\
\hline & IA perifiton & $\begin{array}{l}\text { cl- } a \text { fitoplancton } \\
(\mu \mathrm{g} / \mathrm{L})\end{array}$ & -0.99 & 0 \\
\hline & $\mathrm{SS}(\mathrm{mg} / \mathrm{L})$ & $\mathrm{v}$ media $(\mathrm{m} / \mathrm{s})$ & 0.81 & 0.008 \\
\hline \multirow[t]{3}{*}{$\begin{array}{l}\text { Río } \\
\text { abajo }\end{array}$} & $\mathrm{K}_{\mathrm{d}}(1 / \mathrm{m})$ & $\mathrm{A}_{440}(1 / \mathrm{m})$ & 0.84 & 0.005 \\
\hline & NT (mg/L) & IA perifiton & -0.79 & 0.033 \\
\hline & PT (mg/L) & $\mathrm{A}_{440}(1 / \mathrm{m})$ & 0.75 & 0.021 \\
\hline
\end{tabular}

\section{Correlaciones de las variables estudiadas}

Río arriba, el $\mathrm{K}_{\mathrm{d}}$ se correlacionó de forma positiva con el PT y la $\mathrm{A}_{440}$. Asimismo, el PT $\mathrm{y}$ la $\mathrm{A}_{440}$ se correlacionaron positivamente. El NT se correlacionó de manera positiva con el PS y la cl-a perifítica, mientras que el IA del perifiton se correlacionó de manera inversa con la cl-a fitoplanctónica. Por otro lado, los SS se correlacionaron positivamente con la v (Tabla 2). En el análisis de las variables medidas río abajo se observó que muchas de las correlaciones significativas río arriba, se pierden (Tabla 2). Sólo se conservó la correlación positiva entre el $\mathrm{K}_{\mathrm{d}} \mathrm{y}$ la $\mathrm{A}_{440} \mathrm{y}$ entre el PT y la $\mathrm{A}_{440}$ El NT, por otro lado, mostró una correlación negativa con el IA del perifiton (Tabla 2).

\section{DISCUSIÓN}

Entender los disturbios que las especies invasoras generan es fundamental tanto para la investigación ecológica como para tomar decisiones de manejo, ya que estas especies son uno de los factores principales del cambio ecológico global (Westbrook et al. 2017). La proliferación de diques de castor en los cursos fluviales fueguinos y la ausencia casi total de cursos no impactados en la región elevan la importancia de la investigación de los efectos que los mismos generan (Klotz 2013). En Tierra del Fuego, el enfoque de la investigación en torno a los castores fue cambiando desde el inventario de la población y los impactos ecosistémicos que éstos producen hacia los intentos de su erradicación y la restauración 
de las regiones degradadas (Westbrook et al. 2017). Para llevar a cabo una restauración exitosa, sin embargo, es preciso conocer previamente los impactos fisicoquímicos, biológicos y ecosistémicos que los castores han generado sobre los ríos y arroyos fueguinos.

En estudios previos se encontró que la actividad de castor puede afectar la calidad del agua, aunque la magnitud y la naturaleza de los cambios depende de ciertas características de la cuenca como la geología, tipo y uso del suelo, y el clima (Rosell et al. 2005). Al alterar los niveles de nutrientes, los castores pueden ejercer una influencia considerable en la productividad de las aguas continentales. Naiman y Melillo (1984) encontraron que los diques de castor almacenan aproximadamente mil veces más nitrógeno $(\mathrm{N})$ en los sedimentos que las áreas de rápidos. En arroyos de Quebec también se observó que la fijación de $\mathrm{N}$ puede aumentar aguas abajo de las zonas de influencia del castor vinculado con un aumento en los niveles de PT (Francis et al. 1985). En concordancia con Francis et al. (1985), en el presente estudio, se observó que el PT fue mayor en los sitios con actividad de castor con una tendencia a aumentar en los tramos río abajo. En el caso del NT, si bien río arriba la concentración fue mayor en los sitios con actividad, esta tendencia no se mantuvo en los sitios río abajo. El OD puede reducirse en el agua por la presencia de diques de castor (Naiman et al. 1986). Sin embargo, la concentración se incrementa, por lo general, inmediatamente en la salida del dique, y la reoxigenación completa puede darse dentro de los próximos $250 \mathrm{~m}$ de arroyo. En arroyos de bajo orden no contaminados se espera que no ocurra una falta prolongada de oxígeno por las actividades de los castores (Rosell et al. 2005). En el caso de los sitios estudiados en este trabajo, el OD presentó una tendencia similar a lo esperado, con una concentración levemente mayor en los arroyos sin actividad.

Al disminuir o desviar el flujo de agua, los castores afectan de forma significativa el desplazamiento de la carga de sedimento en los ríos y llanuras de inundación, lo que genera la retención de grandes cantidades de sedimentos. Esta eficiencia de los diques como trampas de sedimento ha sido bien documentada (Naiman et al. 1986, 1988; Butler 1995; Butler and Malanson 1995; Pollock et al. 2007; Burchsted et al. 2010; Westbrook et al. 2011; Westbrook et al. 2013). En consistencia con los trabajos previos, en este estudio se observó que la concentración de SS resultó mayor en los sitios con actividad de castor y disminuyó río abajo de los diques. Del mismo modo, la MOD, estimada como $\mathrm{A}_{440}$, también resultó mayor en los sitios con actividad y disminuyó río abajo. En concordancia con estos resultados, el $\mathrm{K}_{\mathrm{d}}$ también fue mayor en los sitios con actividad, es decir, que la luz se atenúa más rápido en los arroyos con actividad. Estos resultados son esperables debido al efecto de remoción extensiva de los sedimentos que el castor produce para construir los diques (Westbrook et al. 2017).

La generación de diques y el posterior encharcamiento de ríos y arroyos producen cambios significativos en la geomorfología de los cursos de agua (Westbrook et al. 2013). Del mismo modo, tanto el volumen como la velocidad del agua pueden cambiar río abajo, lo que disminuye el caudal anual y atenúa las fluctuaciones estacionales de velocidad (Westbrook et al. 2013). Sin embargo, son pocas las investigaciones que documentaron los efectos de los diques de castor sobre la velocidad y el caudal de los arroyos. Si bien en el presente estudio se encontró que los sitios con actividad de castor presentaron mayores valores de Q y v comparado con los sitios sin actividad, es probable que esta diferencia esté dada por la hidrología natural de los cursos de agua estudiados, más allá de la presencia o ausencia de actividad de castor.

En el agrupamiento por conglomerados río arriba, los sitios que se agruparon más próximos entre sí fueron aquellos con características hidrológicas y morfológicas similares (w y v) y se separaron los sitios sin actividad de castor y los sitios que poseen características marcadamente diferentes al resto. El arroyo Rolito fue el primero en separase, ya que posee características que lo diferencian (más SS y cl-a fitoplanctónica) y pertenece a la región del ecotono. El arroyo Moat, localizado en la región sureste, y con características particulares como mayor concentración de ácidos húmicos y pH ácido, también se separó del resto de los sitios sin formar parte del agrupamiento conjunto. El grupo integrado por el arroyo Hambre, y los ríos Pipo, Lasifashaj y Esmeralda se caracteriza por poseer actividad de castor. Este grupo se separó de los sitios sin actividad (Pampa Alta, Ensenada y Lapataia). Si bien en el análisis de agrupamiento se incluyeron otras variables tales como el PS del perifiton y la clorofila- $a$ perifítica y fitoplanctónica, en el agrupamiento río arriba pudieron diferenciarse dos grupos que se distinguen principalmente por sus 
características hidrológicas y morfológicas y físicas.

Esta tendencia de agrupamiento se vio corroborada con el ACP, en el cual los sitios con actividad se agruparon principalmente por el $\mathrm{w}$ medio y los sitios sin actividad por poseer menor temperatura del agua. Sin embargo, los sitios sin actividad también se agruparon por poseer mayor PS del perifiton, variable asociada al crecimiento de la comunidad y cuya variabilidad fue inversamente proporcional a la temperatura del agua, a diferencia de lo observado en humedales a latitudes más bajas (Rodríguez and Pizarro 2015). Debe tenerse en cuenta que la temperatura del agua es una variable que refleja una medida puntual e instantánea del momento de muestreo y su variabilidad puede estar asociada a factores climáticos pasajeros, especialmente en la zona de estudio, donde, aun durante el verano, se pueden registrar nevadas ocasionales y variaciones marcadas de temperatura asociadas a dicho fenómeno. El PS del perifiton, por otro lado, es una variable más integradora que refleja la biomasa de una comunidad y su estado de desarrollo. Por lo tanto, consideramos que en el caso del agrupamiento de los sitios sin actividad el PS del perifiton tiene una mayor importancia. Así mismo, si bien fue una variable que no tuvo mucho peso en el agrupamiento, los sitios sin actividad poseen menor $\mathrm{K}_{\mathrm{d}}$ lo que implica una mayor penetración de la luz, favoreciendo el desarrollo del perifiton.

En el análisis de agrupamiento de los sitios río abajo los patrones de ordenamiento que se distinguían entre los sitios río arriba se pierden y se forman pequeños grupos no relacionados con la hidrología y morfología de los cursos de agua estudiados. El ACP río abajo también confirmó este patrón. El único grupo que se distingue tanto en el agrupamiento por conglomerados como en el ACP incluye sitios con y sin actividad de castor (río Lasifashaj y arroyos Lapataia y Hambre, en el caso del agrupamiento por conglomerados y ríos Esmeralda y Lasifashaj y arroyos Lapataia y Hambre, en el caso del ACP). Por consiguiente, río abajo de los diques existe una tendencia a la homogeneización de los patrones observados río arriba, probablemente debido a la actividad del castor. Una posible causa de este patrón observado es la disminución de la cobertura del dosel y el consiguiente ensanchamiento de los cauces que esta actividad genera (Anderson and Rosemond 2007). En un estudio previo realizado en arroyos sub-antárticos chilenos también se observó que la actividad del castor produce una homogeneización del microhábitat bentónico asociada al aumento de retención de materia orgánica particulada fina, ocasionada por la generación de diques (Anderson and Rosemond 2007).

A partir de la apertura del dosel, los castores aumentan la superficie de agua sin sombreado, lo que permite que la luz solar llegue a los productores primarios acuáticos. Si esta apertura se extiende aguas abajo de los diques y la luz penetra lo suficiente, la producción primaria debería aumentar río abajo (Klotz 1998). Sin embargo, en el caso de la comunidad perifítica estudiada en este trabajo se observó que la cl-a fue mayor en los sitios sin actividad, mientras que en los sitios con actividad la misma fue mayor río arriba. En contraposición, la cl-a fitoplanctónica resultó mayor (aunque no estadísticamente significativa) en los sitios con actividad. Esto puede explicarse por el aumento de hábitats de aguas estancadas que la actividad de los castores genera en detrimento de las zonas de rápidos. Estas zonas de aguas lentas pueden favorecer a las especies lénticas por sobre las especies lóticas características de estos sistemas (Rosell et al. 2005), generando condiciones favorecedoras de la vida pelágica, que atenúa, a su vez, la luz que llega a los productores primarios bentónicos (Hansson 1992).

Además de alterar la estructura de la comunidad, las especies invasoras modifican la dinámica de la red trófica, ya que alteran la disponibilidad de recursos o pueden dominar el flujo de energía (Vitousek et al. 1987; Strayer et al. 1999). Con la construcción de diques, los castores producen mayor retención de materia orgánica (Naiman et al. 1986) y de nitrógeno (Naiman and Melillo 1984), lo que también genera un aumento de la biomasa de macroinvertebrados (McDowell and Naiman 1986; Anderson and Rosemond 2007). A nivel de las comunidades basales, el efecto de la presencia de castores no evidencia marcados resultados. Si bien son pocos los estudios previos, los mismos reportan que, tanto en su ambiente natural (McDowell and Naiman1986) como en ambientes donde es una especie invasora (Anderson and Rosemond 2007), no se encontraron diferencias en la biomasa perifítica entre sitios río arriba y río abajo de los diques de castor. En este estudio, nuestros resultados evidencian que, independientemente de la presencia de castor, río abajo existe un efecto depresor de 
la biomasa perifítica y fitoplanctónica, y dicho efecto no está relacionado con la concentración de nutrientes, aunque sí se observó que río arriba el NT se correlacionó con la cl-a perifítica.

Según Weber y McFarland (1969) cuando la clorofila- $a$ constituye menos del $1 \%$ de la acumulación natural de materia orgánica perifítica, los organismos heterótrofos constituyen una gran porción del perifiton. El IA del perifiton analizado en este trabajo evidencia una comunidad perifítica altamente heterotrófica en todos los sitios estudiados. Del mismo modo, según Lakatos (1989), el perifiton también resultó de baja biomasa, orgánico y heterotrófico en todos los sitios, independientemente de la presencia de castores. Sin embargo, cabe destacar que en los sitios con actividad el IA fue más del doble que en los sitios sin actividad, evidenciando un aumento de la heterotrofia de la comunidad perifítica producto de la actividad del castor. Esto puede estar relacionado con el aumento de turbidez en el agua y de retención de materia orgánica que el castor genera. A partir de los resultados obtenidos no es posible determinar si el IA aumenta porque aumenta el PSLC o porque la cl- $a$ disminuye, aunque parece ser una combinación de ambos. De todas formas, no es evidente que este aumento esté relacionado con la actividad del castor, sino que puede deberse a las características heterotróficas propias de los ríos y arroyos estudiados y a su baja concentración de nutrientes.

A partir de los resultados obtenidos, podemos concluir que las variables hidrológicas y morfológicas son modificadas por la actividad de castor, lo que queda evidenciado en el agrupamiento de los sitios río abajo de las castoreras, donde las diferencias entre sitios de distintas características hidrológicas y morfológicas se pierden, homogeneizándose. Por otro lado, en estos arroyos naturalmente heterotróficos, el perifiton no demostró ser un buen indicador de la actividad de castor en lo que respecta a las variables de masa. Es posible que la composición algal sea más sensible a los cambios generados por la actividad del castor. Por lo tanto, nuevas investigaciones son necesarias para clarificar el efecto del castor en la composición algal perifítica de los arroyos fueguinos.

AgradeCimientos. Las autoras de este trabajo agradecen a Javier Rojo, Hernán Sacristán, Alex Anzar, María Eugenia López y María Granitto por su colaboración en el trabajo de campo y especialmente a Julio Escobar por asesorarnos sobre los sitios con y sin actividad de castor. También agradecemos el apoyo logístico de Parques Nacionales. El financiamiento para esta investigación fue proporcionado por los proyectos PICT 20151152 y PIDUNTDF a nombre de Patricia Rodríguez, PUECADIC-CONICET 2016 y proyecto GEF GCP/ARG/023/GFF.

\section{REFERENCIAS}

Anderson, C. B., C. R. Griffith, A. D. Rosemond, R. Rozzi, and O. Dollenz. 2006. The effects of invasive North American beavers on riparian communities in Cape Horn, Chile: Do exotic beavers engineer differently in sub-Antarctic ecosystems? Biological Conservation 128:467-474.

Anderson, C. B., and A. D. Rosemond. 2007. Ecosystem engineering by invasive exotic beavers reduces in-stream diversity and enhances ecosystem function in Cape Horn, Chile. Oecologia 154:141-153.

Anderson, C. B., and A. D. Rosemond. 2010. Beaver invasion alters terrestrial subsidies to subantarctic stream food webs. Hydrobiologia 652:349-361.

APHA. 2005. Standard Methods for the Examination of Water and Wastewater. APHA; Washington D.C. Pp. 1268.

Balzarini, M. G., L. González, M. Tablada, F. Casanoves, J. A. Di Rienzo, and C. W. Robledo. 2008. Infostat. Manual del Usuario, Editorial Brujas, Córdoba, Argentina.

Barrera, M. D., J. L. Frangi, L. L. Richter, M. H. Perdomo, and L. B. Pinedo. 2000. Structural and functional changes in Nothofagus pumilio forests along an altitudinal gradient in Tierra del Fuego, Argentina. J Veg Sci 11:179-188.

Burchsted, D., M. Daniels, R. Thorson, and J. Vokoun. 2010. The river discontinuum: applying beaver modifications to baseline conditions for restoration of forested headwaters. BioScience 60:908-922.

Butler, D. R. 1995. Zoogeomorphology: Animals as Geomorphic Agents. Cambridge University Press, New York, USA.

Butler, D. R., and G. P. Malanson. 1995. Sedimentation rates and patterns in beaver ponds in a mountain environment. Geomorphology 13:255-269.

Correll, D. L., T. E. Jordan, and D. E. Weller. 2000. Beaver pond biogeochemical effects in the Maryland coastal plain. Biogeochemistry 49:217-239.

Daciuk, J. 1978. Notas faunísticas y bioecológicas de Península Valdés y Patagonia, IV. Estado actual de las especies de mamíferos introducidos en la Región Araucana (Rep. Argentina) y grado de coacción ejercido en algunos ecosistemas surcordilleranos. Anales de Parques Nacionales 14:105-130. 
Di Rienzo J. A., F. Casanoves, M. G. Balzarini, L. González, M. Tablada, C. W. Robledo. InfoStat versión 2017. Grupo InfoStat, FCA, Universidad Nacional de Córdoba, Argentina. URL: www.infostat.com.ar.

Francis, M. M., R. J. Naiman, and J. M. Melillo. 1985. Nitrogen fixation in subarctic streams influenced by beaver (Castor canadensis). Hydrobiologia121:193-202.

Gordon, N. D., T. A. McMahon, B. L. Finlayson, C. J. Gippel, and R. J. Nathan. 2004. Stream Hydrology: An Introduction for Ecologists. Second edition. Wiley and Sons Ltd., West Sussex, England.

Greenwood, J. L., and A. D. Rosemond. 2005. Periphyton response to long-term nutrient enrichment in a shaded headwater stream. Canadian Journal of Fisheries and Aquatic Sciences 62:2033-2045.

Hansson, L. A. 1992. Factors regulating periphytic algal biomass. Limnology and Oceanography 37:322-328.

Henn, J. J., C. B. Anderson, and G. M. Pastur. 2016. Landscape-level impact and habitat factors associated with invasive beaver distribution in Tierra del Fuego. Biological Invasions 18:1679-1688.

Hill, W. R., and S. E. Fanta. 2008. Phosphorus and light colimit periphyton growth at subsaturating irradiances. Freshwater Biology 53:215-225.

Jespersen, A. M, and K. Christoffersen. 1987. Measurements of chlorophyll- $a$ from phytoplankton using ethanol as extraction solvent. ArchivfürHydrobiologie109:445-454.

Jones, C. G., J. H. Lawton, and M. Shachak. 1994. Organisms as ecosystem engineers. Oikos 69:373-386.

Kirk, J. T. O. 1994. Light and Photosynthesis in Aquatic Ecosystems. Second edition. Cambridge University Press, Cambridge, Reino Unido.

Kirk, J. T. O. 2011. Light and Photosynthesis in Aquatic Ecosystems. Third Edition. University Press, Cambridge, Reino Unido.

Klotz, R. L. 1998. Influence of beaver ponds on the phosphorus concentration of stream water. Canadian Journal of Fisheries and Aquatic Sciences 55:1228-1235.

Klotz, R. L. 2013. Factors driving the metabolism of two north temperate ponds. Hydrobiologia 711:9-17.

Lakatos, G. 1989. Composition of reed periphyton (biotecton) in the Hungarian part of lake Fertö. Biol Forschun Für Burg 71:125-134.

Lazar, J. G., K. Addy, M. K. Welsh, A. J. Gold, and P. M. Groffman. 2014. Resurgent Beaver Ponds in the Northeastern United States: Implications for Greenhouse Gas Emissions J Environ Qual 43:1844-1852.

Lizarralde, M. 1993. Current status of the introduced beaver (Castor canadensis) population in Tierra del Fuego, Argentina. Ambio 22:351-358.

Lowe, R. L., and Y. Pan. 1996. Benthic algal communities as biological monitors. Pp. 705-739 in R. J. Stevenson, M. L. Bothwell and R. L. Lowe (eds.). Algal Ecology: Freshwater Benthic Ecosystems. Academic Press, Londres, England.

Martínez Pastur, G., M. V. Lencinas, J. Escobar, P. Quiroga, L. Malmierca, and M. Lizarralde. 2006. Understorey succession in Nothofagus forests in Tierra del Fuego (Argentina) affected by Castor canadensis. Applied Vegetation Science 9:143-154.

Martínez Pastur, G., M. V. Lencinas, J. M. Cellini, P. L. Peri, and R. Soler Esteban. 2009. Timber management with variable retention in Nothofagus pumilio forests of Southern Patagonia. Forest Ecol Manage 258:436-443.

McDowell, D. M., and R. J. Naiman. 1986. Structure and function of a benthic invertebrate stream community as influenced by beaver (Castor canadensis). Oecologia 68:481-489.

Moore, D. M. 1983. Flora of Tierra del Fuego. Anthony Nelson, UK, Missouri Botanical Garden, St. Louis, USA.

Moorman, M. C., D. B. Eggleston, C. B. Anderson, A. Mansilla, and P. Szejner. 2009. Implications of Beaver Castor canadensis and Trout Introductions on native fish in the Cape Horn Biosphere Reserve, Chile. Transactions of the American Fisheries Society 138:306-313.

Naiman, R. J., C. A. Johnston, and J. C. Kelley. 1988. Alteration of North American streams by beaver. BioScience 38: 753-762.

Naiman, R. J., J. M. Melillo, and J. E. Hobbie. 1986. Ecosystem alteration of boreal forest streams by beaver (Castor canadensis). Ecology 67:1254-1269.

Naiman, R. J., and J. M. Melillo. 1984. Nitrogen budget of a subarctic stream altered by beaver (Castor canadensis). Oecologia 62:150-155.

Pietrek, A. G., and González-Roglich, M. 2015. Post-establishment changes in habitat selection by an invasive species: beavers in the Patagonian steppe. Biological Invasions 17:3225-3235.

Pisano, E. 1981. Bosquejo de la región Fuego-Patagonia. An. Inst. Patagonia 12:159-171.

Pizarro, H., and M. E. Alemanni. 2005. Physical and chemical variables of the water and its influence on the periphytic biomass on the lower parts of the Luján river (Buenos Aires Province). Variables físico-químicas del agua y su influencia en la biomasa del perifiton en un tramo inferior del Río Luján (Provincia de Buenos Aires). Ecología Austral 15(1):73-88.

Pollock, M. M., T. J. Beechie, and C. E. Jordan. 2007. Geomorphic changes upstream of beaver dams in Bridge Creek, an incised stream channel in the interior Columbia River basin, eastern Oregon. Earth Surface Processes and Landforms 32:1174-1185.

Rodríguez, P., and H. Pizarro. 2015. Phytoplankton and periphyton production and its relation to temperature in a humic lagoon. Limnologica-Ecology and Management of Inland Waters 55:9-12.

Rosell, F., O. Bozser, P. Collen, and H. Parker. 2005. Ecological impact of beavers Castor fiber and Castor canadensis and 
their ability to modify ecosystems. Mammal Rev 35:248-276.

Simanonok, M. P., C. B. Anderson, G. Martínez Pastur, M. V. Lencinas, and J. H. Kennedy. 2011. Comparison of impacts from silviculture practices and North American beaver invasion on stream benthic macroinvertebrate community structure and function in Nothofagus forests of Tierra del Fuego. Forest Ecology and Management 262:263-269.

Sokal, R. R., and C. D. Michener. 1958. A Statistical Method for Evaluating Systematic Relationships.

University of Kansas Science Bulletin 38:1409-1438.

Strayer, D. L., N. F. Caraco, J. J. Cole, S. Findlay, and M. L. Pace. 1999. Transformation of freshwater ecosystems by bivalves. BioScience 49:19-27.

Valderrama, J. C. 1981. The simultaneous analysis of total nitrogen and total phosphorus in natural waters. Mar Chem 10:109-122.

Vitousek, P. M., L. R. Walker, L. D. Whitaker, D. Mueller-Dombois, and P. A. Matson. 1987. Biological invasion of Myrica faya alters ecosystem development in Hawaii. Science 238:802-804.

Weber, C. I., and B. H. McFarland. 1969. Periphyton biomass-chlorophyll ratio as an index of surface waters and effluents. Environmental Monitoring Series EPA-670/4-73-001. Cincinnati, Ohio, USA: United States Environmental Protection Agency.

Westbrook, C. J., D. J. Cooper, and C. B. Anderson. 2017. Alteration of hydrogeomorphic processes by invasive beavers in southern South America. Science of the Total Environment 574:183-190.

Westbrook, C. J., D. J. Cooper, and B. W. Baker. 2011. Beaver assisted river valley formation. River Research and Applications 27:247-256.

Westbrook, C. J., D. J. Cooper, and D. Butler. 2013. Beaver geomorphology and hydrology. Pages 293-306 in: D. Butler and C. Hupp (eds.). Treatise on Geomorphology. Academic Press, San Diego, USA.

Wetzel, R. G. 1983. Opening remarks. In R. G. Wetzel (ed.). Periphyton of Freshwater Ecosystems. Dr. W. Junk Publishers, The Hague, Netherlands.

Zuur, A., E. N. Ieno, N. Walker, A. A. Saveliev, and G. M. Smith. 2009. Mixed effects models and extensions in ecology with R. Springer Science and Business Media, New York, USA. 